\title{
Minimal clinically important difference for the 6-min walk test: literature review and application to Morquio A syndrome
}

\author{
Rudolf Schrover ${ }^{1 *}$, Kathryn Evans ${ }^{2}$, Roberto Giugliani ${ }^{3}$, Ian Noble ${ }^{4}$ and Kaustuv Bhattacharya ${ }^{5,6}$
}

\begin{abstract}
Morquio A syndrome is an ultra-rare, inherited lysosomal storage disorder associated with progressive, multisystemic clinical impairments, causing gradual loss of functional capacity and endurance, impaired quality of life, and early mortality. Studies in Morquio A patients have used the 6-min walk test (6MWT) to assess functionality and endurance and to evaluate disease progression or efficacy of treatment. The objective of the present study was to review minimal clinically important differences (MCIDs) for the 6MWT reported for disease states that widely use the $6 \mathrm{MWT}$ to evaluate clinical benefit and to discuss the results in view of the challenges in estimating MCID for ultra-rare diseases, using the case of elosulfase alfa in Morquio A patients. A systematic literature search was performed using Embase and Medline to identify studies specifically estimating the MCID using either anchor-based or distributionbased methods. A total of 19 publications on 17 studies were identified; none of these included patients with Morquio A syndrome or the wider disease category of lysosomal storage disorders. Therefore, the MCIDs determined by studies in patients with respiratory, cardiovascular, or musculoskeletal disease were compared to changes in the 6MWT seen in Morquio A patients in the pivotal phase 3 clinical trial of elosulfase alfa enzyme replacement therapy. The literature review showed a mean MCID for the 6MWT of 7\% change (range 3-15\%) in studies using anchor-based methods and a $9 \%$ change (range 4-16\%) using distribution-based methods. Results of the elosulfase alfa clinical trial and its extension showed a placebo-adjusted $14.9 \%$ improvement in the 6MWT from baseline at week 24, which was greater than the mean MCID based on the results of the systematic literature review. After 2 years, 6MWT distance increased by a mean of $20.7 \%$ from baseline in a modified per-protocol population, versus a reduction of $6.9 \%$ in comparable untreated patients from the MorCAP natural history study over the same period. Although further research is required to establish the MCID of the 6MWT in Morquio A patients, the presented data provide further evidence for the positive effect of elosulfase alfa in this patient population.
\end{abstract}

Keywords: Enzyme replacement therapy, Morquio A syndrome, Mucopolysaccharidosis IVA, Endurance, Six-minute walk test, Minimal clinically important difference, MPS IVA

\section{Background}

Morquio A syndrome, also known as mucopolysaccharidosis (MPS) IVA, is an ultra-rare, severely debilitating and progressively life-threatening inherited disorder (ORPHA309297) [1]. It is caused by mutations of the gene $\mathrm{N}$-acetylgalactosamine-6-sulfatase (GALNS), resulting in impaired degradation of the glycosaminoglycans (GAGs) keratan sulfate (KS) and chondroitin-6-sulfate

\footnotetext{
* Correspondence: rschrover@synevi.com

'SYNEVi Pty Limited, Level 4, Suite 402, 15 Help Street, Chatswood NSW 2067, Australia

Full list of author information is available at the end of the article
}

(C6S). As a result, KS and C6S accumulate in the lysosomes of cells throughout the body, which leads to widespread cellular, tissue, and organ dysfunction [1-4].

All patients with Morquio A syndrome suffer from a range of serious and debilitating morbidities across multiple domains including skeletal and joint abnormalities, short stature, impairments in respiratory function (restrictive and obstructive lung disease), cardiac function (valvular regurgitation and stenosis, low stroke volume), hearing, and vision, abdominal manifestations (hepatomegaly, hernias), and dental abnormalities, whilst retaining normal intelligence [1, 5-9]. The 
significant limitations in respiratory function, cardiac function, and musculoskeletal compromise result in low endurance, debilitating fatigue, pain, poor quality of life, and early mortality $[4,5,10]$. Due to low endurance and functional capacity limitations, many patients with Morquio A syndrome end up using a wheelchair or walking aid [1]. Historically, survival in patients with Morquio A syndrome has been severely reduced with the majority of patients dying in their second or third decade of life, mainly due to cardiorespiratory failure [4]. Less than 5\% of patients live beyond 40 years [5].

Impaired endurance and functional capacity, both of which are important patient-relevant outcomes for Morquio A patients, may be measured clinically through the six-minute walk test (6MWT), which is a simple, commonly used, standardized measure of endurance [11]. It evaluates the global and integrated responses of all body systems involved during exercise, including the pulmonary and cardiovascular systems, systemic and peripheral circulation, and neuromuscular function. The American Thoracic Society (ATS) Consensus Statement describes the validated approach for performing the 6MWT [11].

Baseline data from the Morquio A Clinical Assessment Program (MorCAP) study, a longitudinal natural history study examining the outcomes of patients with Morquio
A, has shown that endurance and functional capacity, as measured by the 6MWT, are severely impaired in patients with Morquio A syndrome [4]. The overall mean ( \pm SD) 6 MWT distance of $212 \pm 152 \mathrm{~m}$ in MorCAP was significantly lower than that of healthy individuals aged 4-16 years, reported as between 470 and $664 \mathrm{~m}[12,13]$. Moreover, a cross-sectional analysis showed that the mean 6MWT distance generally decreases with increasing age in patients with Morquio A syndrome: mean 6MWT distance was $251.6 \mathrm{~m} \pm 121.5 \mathrm{~m}$ in $0-4$ year olds $(n=37), 232.5 \mathrm{~m} \pm 140.1 \mathrm{~m}$ in $5-11$ year olds $(n=127)$, $181.2 \mathrm{~m} \pm 177.3 \mathrm{~m}$ in $12-18$ year olds $(n=84)$, and $193.1 \mathrm{~m} \pm 148.5 \mathrm{~m}$ in 18 year olds $(n=68)$, thus illustrating the progressive nature of the disease [4]. This progressive decline in 6MWT is further illustrated in Fig. 1, comparing untreated Morquio A subjects with the normal population $[4,14]$. Further evidence that untreated Morquio A syndrome is progressive and characterized by a gradual decline in endurance has been provided by 1 - and 2-year data from MorCAP, in which a general decline in 6MWT distance from baseline was observed over the course of the 2-year longitudinal study [15]. At each visit, mean 6MWT distances were found to generally decrease with increasing age with an annualized estimate of change (SE) in 6MWT from baseline of $-4.86 \pm 3.25 \mathrm{~m}$ across all subjects, and $-6.84 \pm 5.38 \mathrm{~m}$

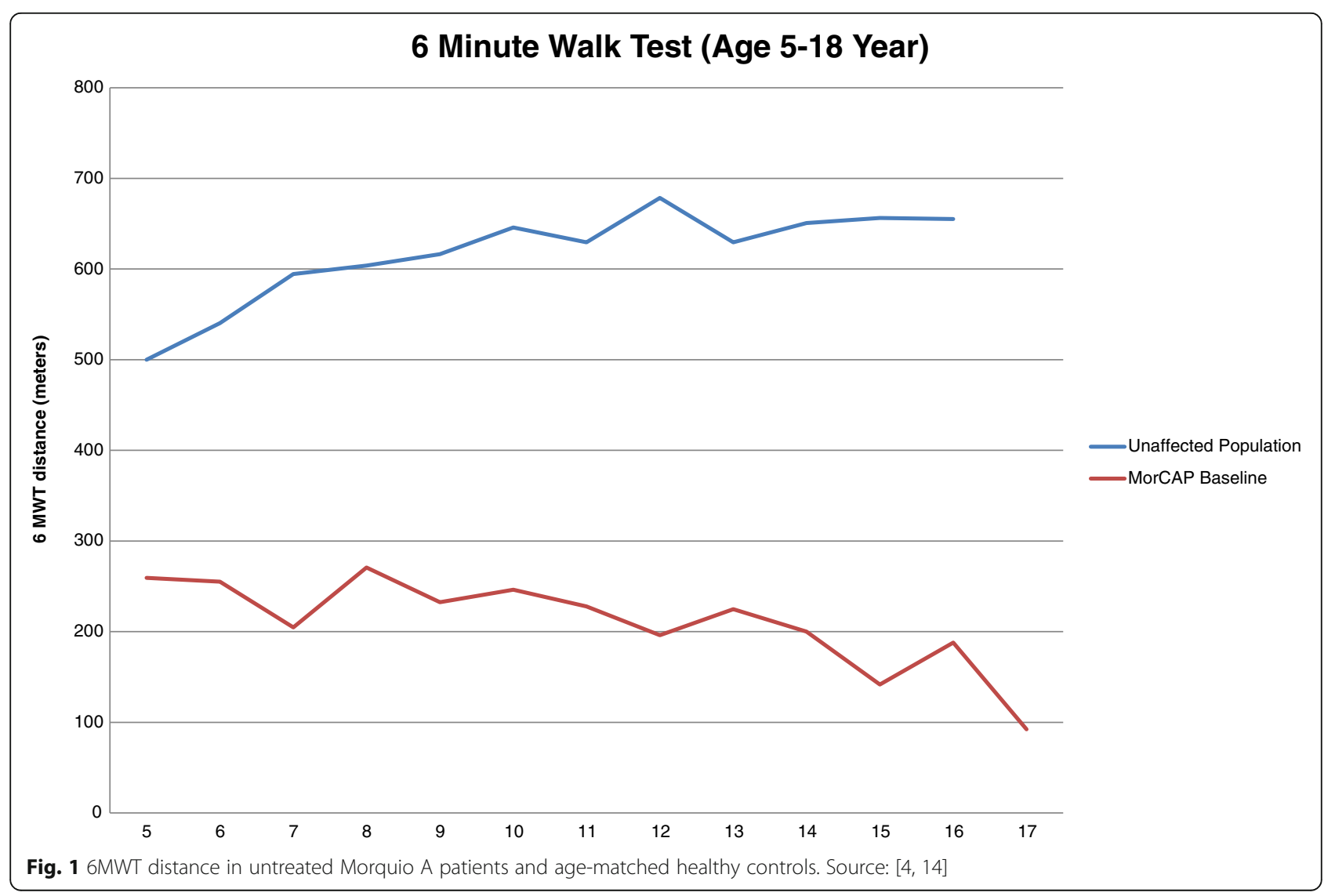


for subjects meeting the inclusion/exclusion criteria of the Phase 3 clinical trial of elosulfase alfa ( $\geq 5$ years of age with baseline 6MWT distance $\geq 30$ and $\leq 325 \mathrm{~m}$ ) [15]. For these reasons, the 6MWT was the primary endpoint in the pivotal Phase 3 randomized, doubleblind, placebo-controlled MOR-004 clinical trial of elosulfase alfa enzyme replacement therapy (ERT). In this study, a statistically significant improvement of $22.5 \mathrm{~m}$ (95\% CI 4.0, 40.9; $p=0.017$ ) in 6MWT distance over placebo was demonstrated with elosulfase alfa $2.0 \mathrm{mg} /$ $\mathrm{kg} /$ week at 24 weeks [10], which was sustained over 120 weeks [16]. This incremental change in the $6 \mathrm{MWT}$ represents a $14.9 \%$ placebo-adjusted change from baseline at 24 weeks (Additional file 1). At 2 years in the open-label extension trial MOR-005, 6MWT distance increased by a mean of $11.7 \%$ (SD 57.2) versus baseline in the Intent-to-treat (ITT) population and a mean of 20.7\% (SD 57.0) versus baseline in the Modified-PerProtocol (MPP) population (excluding patients who had orthopedic surgery during the extension study or missed $\geq 20 \%$ of their scheduled elosulfase alfa infusions), versus decreases of $7.2 \%$ (SD 35.7) and 6.9\% (SD 32.7) over the same period in comparable untreated patients from the MorCAP natural history study (Additional file 2). In addition to the effects on $6 \mathrm{MWT}$, long-term (2 years) treatment with elosulfase alfa was associated with a sustained reduction of around $60 \%$ in urinary $\mathrm{KS}$, a mean increase of 6.8 stairs/min in the 3-min stair climb test (3MSCT), improvements in respiratory function $(+9.2 \%$ for forced vital capacity, $8.8 \%$ for forced expiratory volume in $1 \mathrm{~s}$, and $6.1 \%$ for maximum voluntary ventilation), and improvements in the patients' abilities to perform activities of daily living (MPP population) [16-18].

A recent study by Lampe et al. [19] showed an association between 6MWT and quality of life in patients with Morquio A syndrome. The authors reported that an increase of $100 \mathrm{~m}$ in 6MWT distance was associated with a 0.2 increase in the quality of life utility score of the EuroQoL 5 domains, 5 levels (EQ-5D-5 L) questionnaire. There are currently no studies reporting the relationship between the 6MWT and survival in patients with Morquio A syndrome. However, many studies in widely varying diseases show a positive correlation between the $6 \mathrm{MWT}$ and survival, especially in diseases associated with compromised respiratory and cardiovascular function [20-26]. As Morquio A syndrome is associated with multi-systemic clinical impairment and the most common causes of death are due to cardiorespiratory failure, it is logical to assume from a biological perspective that the relationship between the $6 \mathrm{MWT}$ and survival is also present in patients with Morquio A syndrome.

Although an improvement or decline in the 6MWT distance seems important as such, the clinical relevance and impact on patients' daily functioning of changes in the 6MWT distance observed in clinical trials remains to be established for Morquio A. A standard way of measuring clinical relevance is determining the minimal clinically important difference (MCID), which is the smallest difference that a patient would perceive as beneficial [27]. The objective of this study was to review published studies which aimed to estimate the MCID for the 6MWT in a variety of disease states that widely use the $6 \mathrm{MWT}$ to evaluate clinical benefit and to discuss the results in view of the challenges of estimating MCID in ultra-rare diseases using the case of elosulfase alfa in Morquio A patients.

\section{Methods}

\section{A systematic review of the literature}

Literature searches were conducted in April 2014 using Embase with data covering both Embase and Medline. The search terms used included all variations of 'sixminute walk test' (e.g., 'walking test'; ' 6 min walk test', or '6MWT') and all forms of the term 'minimal clinically important difference' including 'MCID'; 'clinically important', or 'clinically significant'. The search was not restricted to Morquio A syndrome in order to identify studies in other diseases that could help interpret the clinical relevance of 6MWT as an outcome measure. Furthermore, the search was also not limited by study type or language. The retrieved articles were reviewed to identify studies that reported a threshold for the 6MWT that was considered clinically meaningful. The reference lists of all retrieved papers were reviewed for any additional studies that did not appear in the original literature search. The results for each of the identified studies were extracted and grouped according to the MCID estimation method used.

There are three general approaches for estimating the MCID: anchor-based, distribution-based, and consensusbased methods. These approaches measure a quantifiable change in the 6MWT. Anchor-based methods compare the change in a clinical measure to the change in a patient-related anchor such as a patient-reported outcome (PRO), typically a global assessment rating in which the patients rate themselves as "better", "unchanged", or "worse" [28]. Some studies have used death, hospitalization, or other clinical measures (e.g., FVC) as "anchors" [29-32]. Within the anchor-based approaches, four variations exist including the withinand between-patients score change as well as sensitivity and specificity approaches and the social comparison approach [28]. Distribution-based methods compare the change in an outcome to a standardized measure of variability such as the standard error of measurement (SEM), standard deviation (SD), effect size, or minimum detectable change (MDC). Full details on 
variations of the anchor- and distribution-based methods are described in a review by Copay A G, et al. [33]. Consensus-based methods, also called Delphibased methods, use expert panel opinion to establish which numerical value constitutes the minimal clinically relevant change for an outcome measure [34].

\section{Results and discussion}

A total of 19 publications on 17 studies which aimed to estimate the MCID for the 6MWT in diseases other than Morquio A syndrome were identified (Fig. 2). These comprised three broad categories, including respiratory disease (idiopathic pulmonary fibrosis [IPF], diffuse parenchymal lung disease, chronic obstructive pulmonary disease [COPD], non-cystic fibrosis bronchiectasis, emphysema), cardiovascular disease (chronic heart failure $[\mathrm{CHF}]$, coronary artery disease $[\mathrm{CAD}]$, pulmonary arterial hypertension $[\mathrm{PAH}])$, and muscular disease (Duchenne muscular dystrophy [DMD]) (Table 1). One study was conducted in frail elderly Asian persons and was allocated to the category "Other". Although no studies in Morquio A syndrome were identified, the disease categories are characteristic of the symptoms commonly experienced by patients with Morquio A syndrome. A total of 52 estimates of the $6 \mathrm{MWT}$ MCID were reported of which $40 \%$ (21/52) were determined using an anchor-based method approach while
$52 \%(27 / 52)$ were estimated using a distribution-based method. In four cases (8\%), the method to calculate MCID was not reported.

\section{Absolute 6MWT MCID}

The results of the studies estimating the MCID for the $6 \mathrm{MWT}$ using the anchor-based approach are presented in Fig. 3. The MCIDs as measured in absolute terms of meters walked ranged from 11-54 $\mathrm{m}$ across all studies. When considered separately by respiratory, cardiovascular, muscular, and other diseases, the MCID ranges were 11-54 m, 23-45 m, $26 \mathrm{~m}$, and $18 \mathrm{~m}$, respectively.

The results of the studies estimating the MCID for the 6MWT using various distribution-based methods are presented in Fig. 4. Several different variations of the distribution-based methods were reported, including SEM, effect size, and 'other methods'. The category 'other methods' are those studies that used variants of the standard deviation approach as well as those studies that did not clearly report the exact distribution-based method used. The range of MCIDs for the 6MWT using the SEM, effect size, and 'other methods' was similar to that seen in studies using the anchor-based approach with ranges between 13 and $45 \mathrm{~m}$ (excluding an $80 \mathrm{~m}$ outlier), 20-59 m, and 29-47 m, respectively. When the estimated MCIDs were considered by respiratory, cardiovascular, and 'other' diseases, the results were also

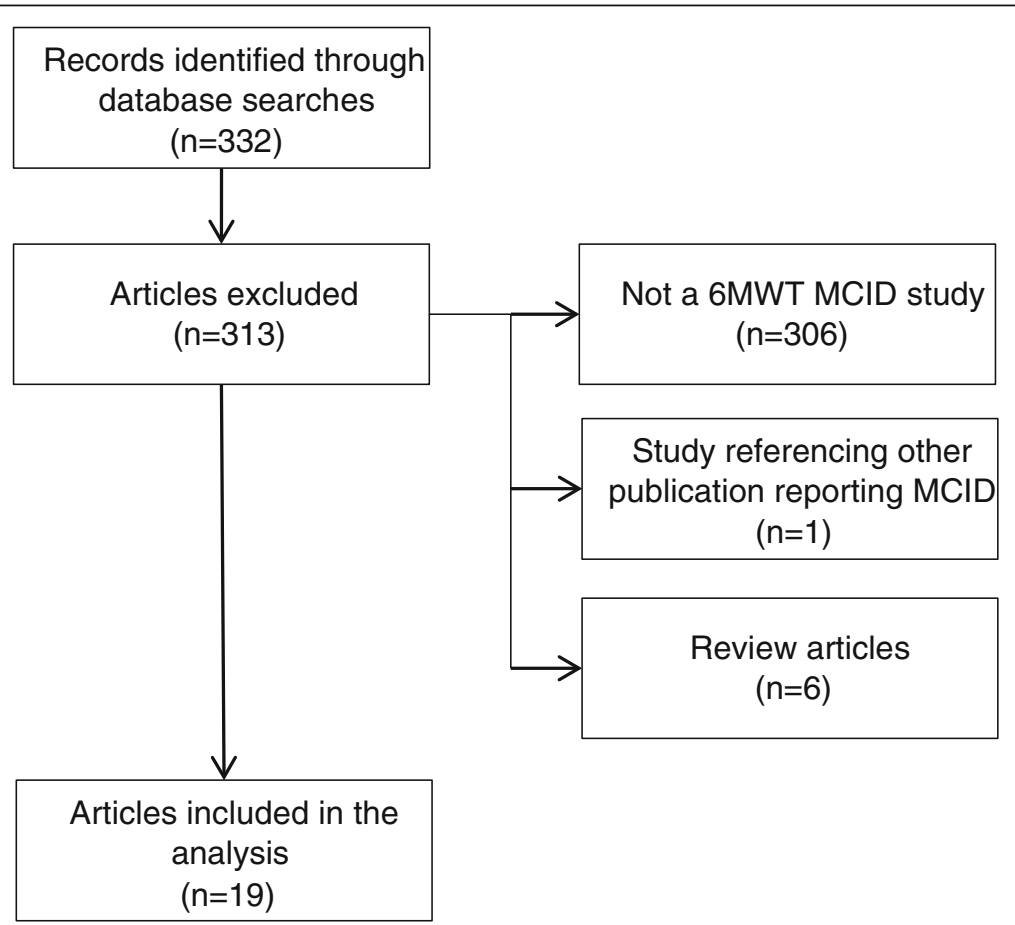

Fig. 2 Flow chart summary of the systematic literature search. Studies that aimed to estimate the minimal clinically important difference (MCID) for the six-minute walk test (6MWT) in diseases relevant to Morquio A syndrome including respiratory, cardiovascular, and muscular diseases were included 
Table 1 Overview of studies that estimate the minimal clinically important difference (MCID) for the six-minute walk test (6MWT)

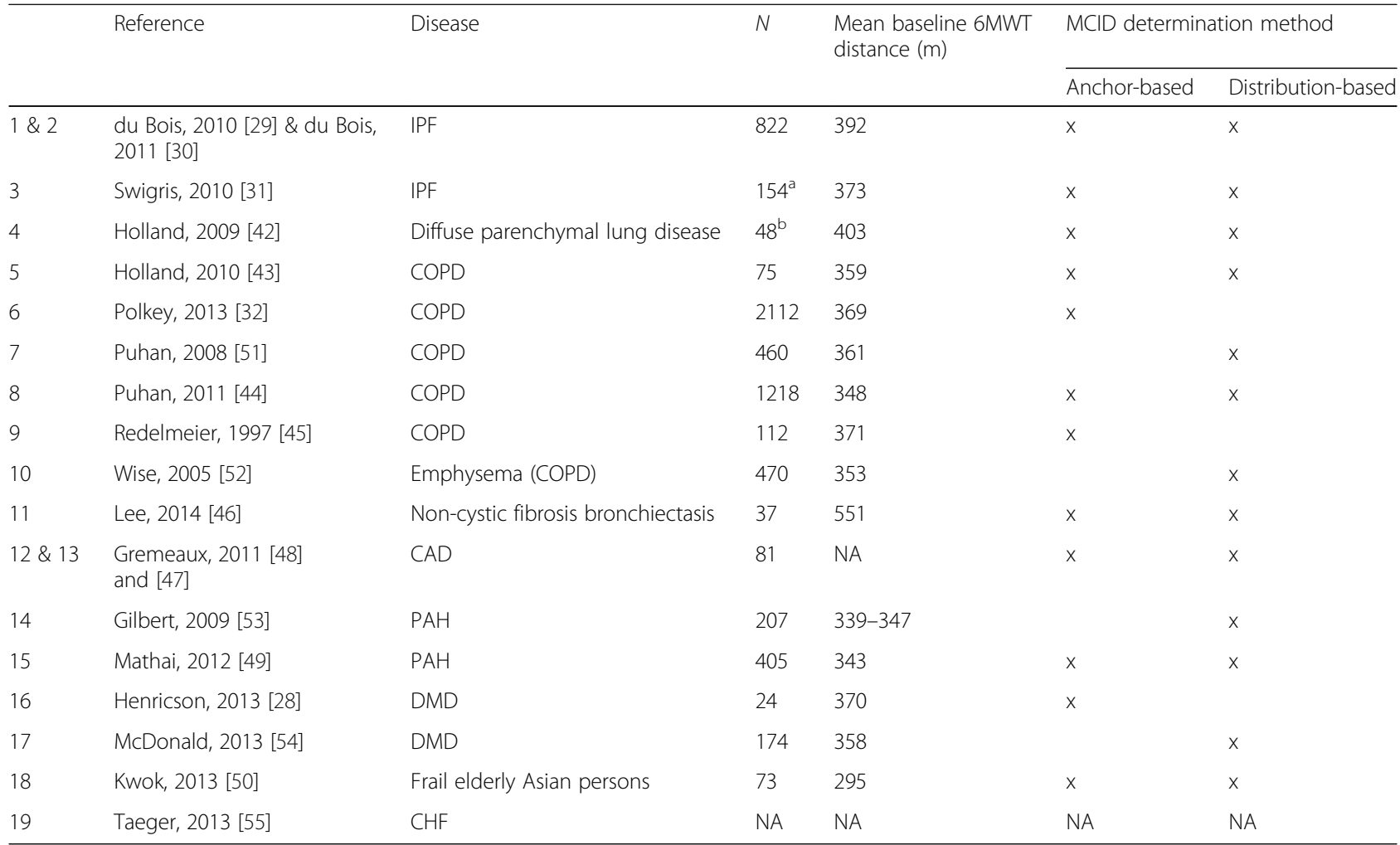

CAD coronary artery disease, $C H F$ chronic heart failure, COPD chronic obstructive pulmonary disease, DMD Duchenne muscular dystrophy, IPF idiopathic pulmonary fibrosis, $M C I D$ minimal clinically important difference, $N A$ not available, $P A H$ pulmonary arterial hypertension

${ }^{a}$ Only 123 patients had data at baseline and follow-up

b24 patients had IPF

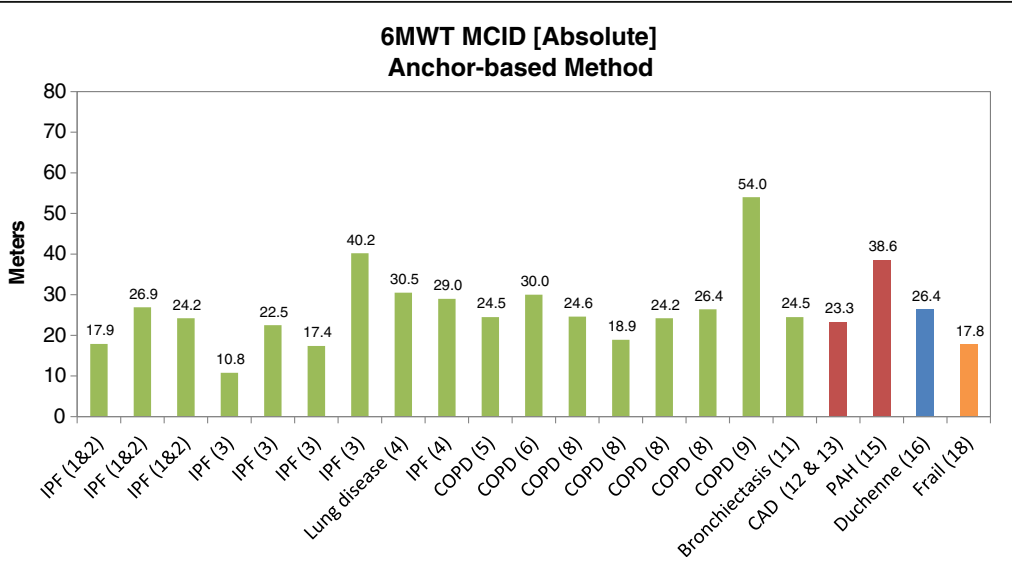

Fig. 3 Absolute six-minute walk test (6MWT) minimal clinically important difference (MCID) using anchor-based methods. Sources: 1 \& $2=$ du Bois, 2010 [29] and du Bois, 2011 [30]; 3 =Swigris, 2010 [31]; 4 = Holland, 2009 [42]; $5=$ Holland, 2010 [43]; $6=$ = Polkey, 2013 [32]; $8=$ Puhan, 2011 [44]; 9 = Redelmeier, 1997 [45]; 11 =Lee, 2014 [46]; 12 \& 13=Gremeaux, 2011 [47] and Gremeaux, 2011 [48]; $15=$ Mathai, 2012 [49]; $16=$ Henricson, 2013 [28]; 18 = Kwok, 2013 [50]. The green, red, blue, and orange bars represent studies conducted in patients with respiratory, cardiovascular, muscular, and 'other' (i.e., frail elderly) diseases, respectively. CAD: coronary artery disease; COPD: chronic obstructive pulmonary disease; IPF: idiopathic pulmonary fibrosis; PAH: pulmonary arterial hypertension 


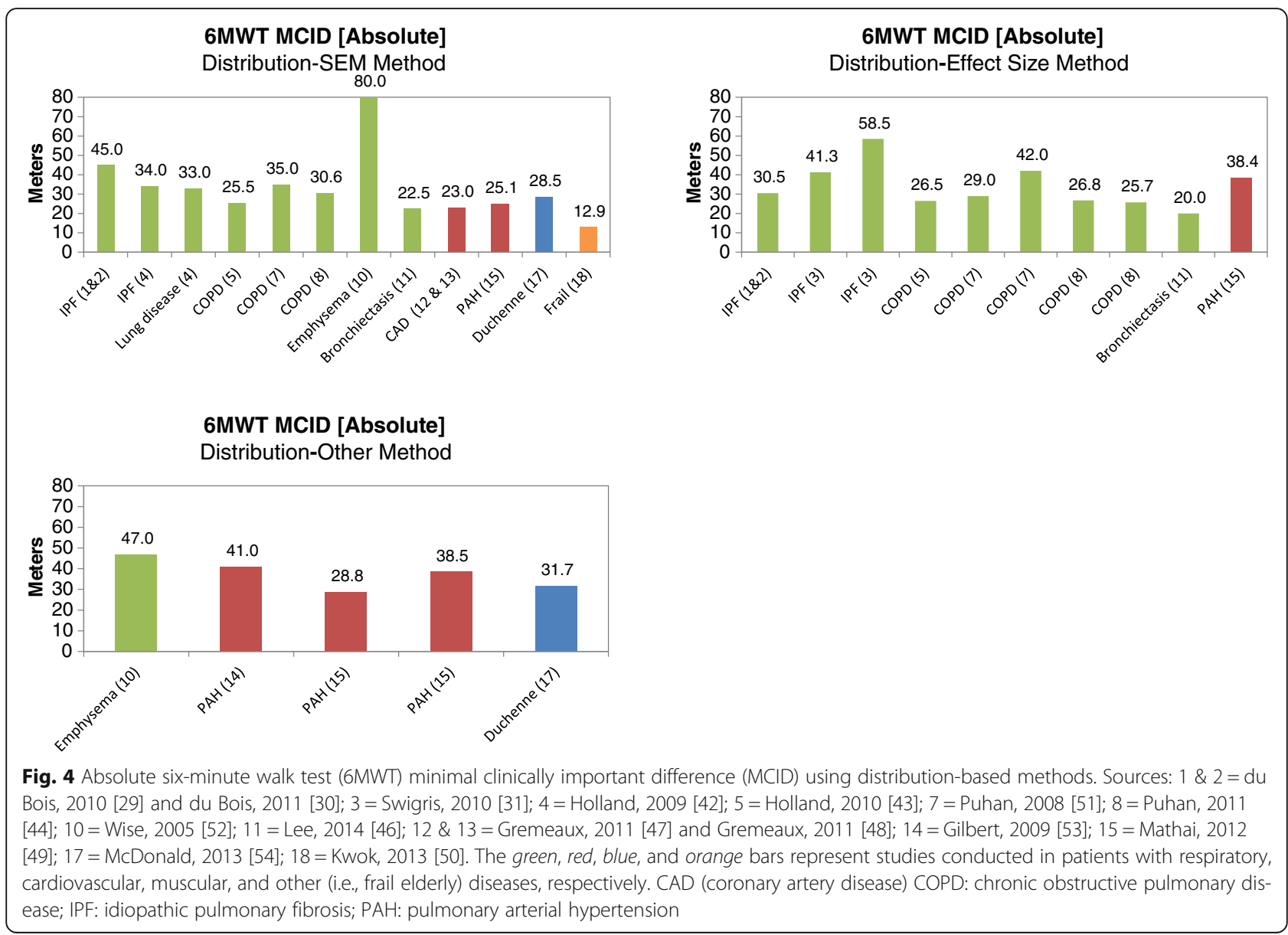

similar. Although the absolute measures of the MCID for the $6 \mathrm{MWT}$ are informative, there are notable differences in terms of ability to walk between the patients enrolled in the studies considered in this analysis and those with Morquio A syndrome. The distance walked in the 6MWT at baseline in the MorCAP study was $212.6 \pm 152.2 \mathrm{~m}$ [4], which is much lower than the average of $375 \mathrm{~m}$ for the clinical intervention studies included in this analysis. Note, however, that baseline $6 \mathrm{MWT}$ results were not reported for all included studies. Additionally, the influence of short stature on distance walked has not been estimated in any study, whereas the median Z-score for height for adults with Morquio A syndrome in the MorCAP study was -8.94 [4]. This severe short stature undoubtedly influences the total distance walked meaning that relative rather than absolute changes in distance walked are more indicative of therapy benefit compared with other studies.

In most studies, the absolute MCID was calculated without any specific intervention, so it can be considered as a measure of disease burden, with smaller 6MWT MCIDs representing a greater disease burden.

\section{Relative 6MWT MCID}

In the absence of published studies reporting an MCID for Morquio A syndrome, it may be considered appropriate to view the MCID in relative change rather than absolute change from baseline to help interpret the clinical relevance of $6 \mathrm{MWT}$ changes. Therefore, the MCID was estimated as the required percentage change in meters from baseline for those studies reporting the results of the 6MWT at baseline. Since not all studies reported the results of the 6MWT at baseline, the number of studies included in the analysis was reduced to 14 studies reporting a total of 40 estimates of 6MWT MCID.

The results of the re-analyzed MCID studies using the anchor-based method are presented in Fig. 5. The MCID for the $6 \mathrm{MWT}$ as measured in relative terms is a mean of $7 \%$ (range $3-15 \%$ ). When considered separately by respiratory, cardiovascular diseases, muscular disease, and 'other' the results were $3-15,11,7$, and $6 \%$, respectively.

The relative re-analyzed MCID for the 6MWT estimated using the various distribution-based methods are presented in Fig. 6 . The mean relative change using this method is $9 \%$ with a range of $4-16 \%$ (excluding a $23 \%$ outlier) across all diseases. When the relative MCIDs 
6MWT MCID [Relative]

Anchor-based Method

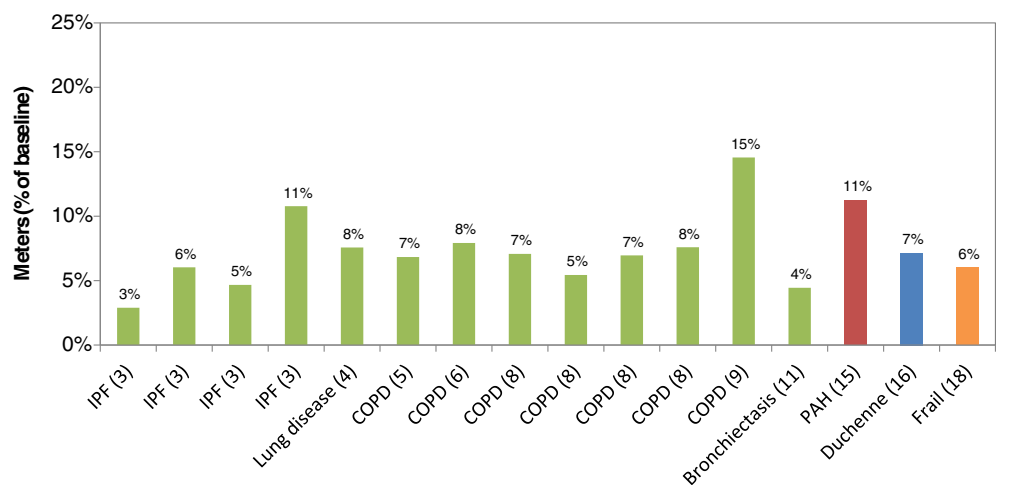

Fig. 5 Relative six-minute walk test (6MWT) minimal clinically important difference (MCID) using Anchor-based methods. Sources: $3=$ Swigris, 2010 [31]; 4 = Holland, 2009 [42]; 5 = Holland, 2010 [43]; 6 = Polkey, 2013 [32]; $8=$ Puhan, 2011 [44]; $9=$ Redelmeier, 1997 [45]; $11=$ Lee, 2014 [46]; $15=$ Mathai, 2012 [49]; 16 = Henricson, 2013 [28]; 18 = Kwok, 2013 [50]. The green, red, blue, and orange bars represent studies conducted in patients with respiratory, cardiovascular, muscular, and other (i.e., frail elderly) diseases, respectively. COPD: chronic obstructive pulmonary disease; IPF: idiopathic pulmonary fibrosis; PAH: pulmonary arterial hypertension

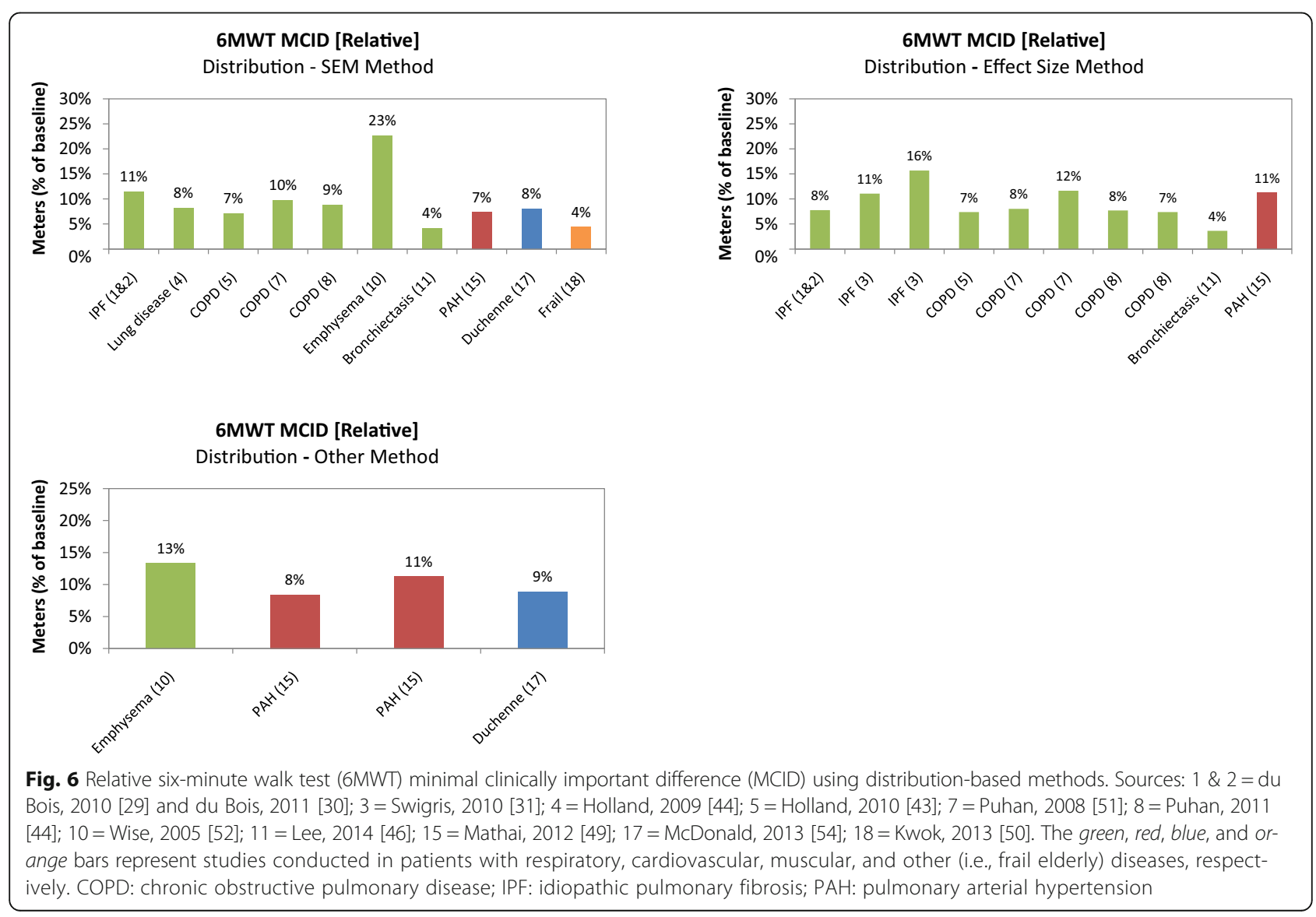


were considered by respiratory, cardiovascular, muscular, and 'other' diseases, the results were 4-16, 7-11, 8-9, and $4 \%$, respectively.

\section{Applying MCIDs from other chronic diseases to Morquio A syndrome}

The average relative MCID for the 6MWT in other chronic diseases is similar in anchor-based (mean 7\%; range 3-15\%) and distribution-based methods (mean 9\%; range 4-16\%). In the absence of any specific published studies on the MCID for the 6MWT in Morquio A syndrome, these values may serve as an evidencebased proxy until an MCID using 6MWT or other relevant clinical or patient-reported measures for Morquio A syndrome is established.

The mean 6MWT distances at baseline in the Phase 3 randomized, double-blind, placebo-controlled clinical trial of elosulfase alfa (MOR-004) were 203.9 and $211.9 \mathrm{~m}$ for patients treated with elosulfase alfa and placebo, respectively. At week 24, the estimated treatment effect compared with placebo was $22.5 \mathrm{~m}$, corresponding with a change from baseline of $14.9 \%$. The mean percentage improvement from baseline was $23.9 \%$ and $8.7 \%$ for patients treated with elosulfase alfa $2.0 \mathrm{mg} / \mathrm{kg} /$ week and placebo, respectively. In the long-term MOR-005 extension study, the mean change from baseline after 2 years in the 6MWT was $11.7 \%$ in the ITT and $20.7 \%$ in the MPP population, versus a reduction of $7.2 \%$ and $6.9 \%$, respectively in comparable untreated patients from the MorCAP natural history study [16]. The observed treatment difference is greater than the $7-9 \%$ described above and greater than or at the upper limit of the range of MCIDs calculated for other diseases using anchorbased and distribution-based methods. Of note, one of the inclusion criteria of the MOR-004/005 trial was a baseline 6MWT distance $\geq 30$ and $\leq 325 \mathrm{~m}$ [10]. However, not all Morquio A patients are ambulatory. The value of the 6MWT is not maintained in case of walking difficulties due to limb deformities, even when the patient has a relatively good muscular and cardiovascular state.

It is important to qualify the limitations of applying the MCID for the 6MWT of other chronic diseases to Morquio A syndrome. Specifically, patients with Morquio A syndrome experience respiratory, cardiovascular, and musculoskeletal impairment and as such their ability to perform the 6MWT is more severely compromised compared with those enrolled in studies investigating the MCID of the 6MWT in only one body system (e.g., respiratory, cardiovascular). While the above-mentioned range of relative MCIDs for other chronic diseases may provide an indication of the 6MWT MCID in Morquio A syndrome, it is likely that the true MCID for the $6 \mathrm{MWT}$ in patients with Morquio A syndrome is lower.
As patients with Morquio A syndrome tend to have a lower functional capacity at baseline than the other diseases included in the present study, smaller improvements in 6MWT distance might be clinically important, as previously demonstrated for patients with DMD with varying baseline $6 \mathrm{MWT}$ results [35]. The analysis also does not take into account the age of patients included in the different studies, which range from young children of 4-12 years (DMD studies) to elderly patients of around 60-70 years of age for most respiratory diseases. This might, at least partly, explain the large differences in baseline 6MWT distances between studies. The age of the Morquio A patients included in the MOR-004/005 studies ranged from 5-57 (mean 14.4) years. In addition, the relevance of 6MWT MCIDs for the patients' healthrelated quality of life (HRQoL) in the chronic diseases included in the present analysis is unclear [36]. The 6MWT is generally not used as a measure of HRQoL for these conditions. Although 6MWT distance has been found to be consistently associated with symptoms such as peak work capacity and physical activity in these conditions, correlations with HRQoL are generally weak [37]. For Morquio A, a sub-analysis of data from a PRO study $(N=24)$ recently showed a strong correlation between 6MWT distances and EuroQoL (EQ)-5D-5 L utility scores $(R=0.815 ; P<0.0001)$ [19]. However, the association between changes in the 6MWT and HRQoL needs to be established. Of note, several pivotal placebo-controlled clinical trials in patients with lysosomal storage disorders similar to Morquio A (i.e., MPS I, MPS VI, and late-onset Pompe disease) have used the $6 \mathrm{MWT}$, or the comparable 12MWT, as a primary endpoint to determine treatment efficacy for the ERT being assessed [38, 39]. Regulators and payers agreed that the 6MWT outcomes provided enough evidence for a clinically relevant treatment effect for these diseases leading to the approval and reimbursement of these medications for treating the designated disorders. The fact that the long-term improvements in the 6MWT in Morquio A patients in the MOR-004/005 study were accompanied by improvements in respiratory function and the ability of patients to perform activities of daily living also provides further evidence for the clinical relevance of $6 \mathrm{MWT}$ results in these patients $[17,18]$. It remains to be elucidated whether the improvements in respiratory function contribute to the improvements in 6MWT outcomes. Other mechanisms, such as increases in muscle strength, joint movement, or pain may also be involved.

Further research is required to directly establish the MCID of the 6MWT in patients with Morquio A syndrome as a reliable measure of treatment outcome. However, this entails selection of the most appropriate method first, taking into account the limitations of 
currently used MCID determinations. As illustrated by the wide distribution in MCIDs between the studies included in the present analysis, often within the same diseases state, different analysis methods produce different MCIDs. Whereas anchor-based methods yield different MCIDs depending on the PRO scale used and the arbitrary selection of grouping of scale levels, MCIDs obtained with distribution-based methods depend on the measure of statistical variability used in the analysis [33]. Another limitation of anchor-based methods is that the PRO measures used to calculate MCIDs are subjective, often not validated for the disease, and subject to recall bias [33]. In addition, PRO changes generally depend on baseline level, with patients with greater level of disability showing more improvement. Major issues of distribution-based methods are that outcomes are sample-specific, i.e., depend on the variability of results in the sample studied, and do not address the question of clinical importance for the patient [33].

\section{Conclusions}

The identification of clinically relevant outcomes is critical in the evaluation of the efficacy of therapeutic interventions. From a physician's, but also a payer's and regulator's perspective, it is important to understand the medical consequences of a treatment and the limitations of the disease to properly assess clinical improvements. Ideally each disease should have its own MCID determined. However, establishing evidence of clinical effectiveness can be very challenging for ultra-rare diseases such as Morquio A syndrome due to the very small number of physicians with specialized expertise in the disease, limited understanding of the natural history, and difficulties in generating a large volume of evidence in randomized clinical trials [40]. In the absence of sufficient data, it can be valuable to compare outcomes in studies of ultra-orphan diseases with MCIDs for that outcome established for other, better characterized conditions (respiratory, cardiovascular, and muscular diseases). Applying this strategy to the 6MWT in Morquio A provided further evidence for the clinical relevance of elosulfase alfa ERT as a treatment option. The mean MCID for the 6MWT of $7-9 \%$ change identified in studies using anchor-based and distribution-based methods is considerably below the $14.9 \%$ improvement over placebo at week 24 in Morquio A patients treated with elosulfase alfa in the pivotal phase 3 study. This improvement was sustained with long-term treatment. However, as this approach is subject to several limitations, the next step is to directly establish the MCID of the 6MWT in patients with Morquio A syndrome. Considering the advantages and limitations of currently used MCID determinations [33], both anchor- and distribution-based methods should ideally be used. A
PRO measure that has been used successfully in Morquio A patients, and might therefore be useful when establishing the MCID for the 6MWT in Morquio A patients using an anchor-based method, is the MPS Health Assessment Questionnaire (MPS-HAQ), an MPS-specific questionnaire that assesses the patient's ability to perform activities of daily living $[1,41]$. Other, generic, PRO measures that have been proven useful in these patients are the EQ-5D questionnaire (assessing mobility, self-care, usual activities, pain/discomfort, and anxiety/depression), and the Brief Pain Inventory Short Form (BPI-SF) and the Adolescent Pediatric Pain Tool (APPT) that assess pain intensity in adults and children/adolescents, respectively [1].

\section{Additional files}

Additional file 1: Treatment effect estimates for ANCOVA of 6-min walk test (6MWT) percent change from baseline to week 24. Analysis population: Intent-To-Treat. (DOCX 12 kb)

Additional file 2: Percent change from MOR-004 baseline in 6-min walk test (6MWT) distance at 2 years in the MOR-005 Modified Per-Protocol (MPP) population (excluding patients who had orthopedic surgery during the extension study or missed $\geq 20 \%$ of their scheduled elosulfase alfa infusions) and comparable, untreated patients from the MorCAP natural history study. (DOCX $12 \mathrm{~kb}$ )

\section{Abbreviations}

6MWT: 6-min walk test; APPT: Adolescent Pediatric Pain Tool; BPI-SF: Brief Pain Inventory Short Form (BPI-SF); CAD: Coronary artery disease; CHF: Chronic heart failure; COPD: Chronic obstructive pulmonary disease; DMD: Duchenne muscular dystrophy; ERT: Enzyme replacement therapy; GAGs: Glycosaminoglycans; GALNS: N-acetylgalactosamine-6-sulfatase; IPF: Idiopathic pulmonary fibrosis; MCID: Minimal clinically important difference; MorCAP: Morquio A Clinical Assessment Program; MPS: Mucopolysaccharidosis; MPS-HAQ: MPS Health Assessment Questionnaire; NA: Not available; PAH: Pulmonary arterial hypertension; PRO: Patient-reported outcomes

\section{Acknowledgements}

The authors are grateful to Brad Dalton of MedScript Communications and Ismar Healthcare NV who provided medical writing and editorial assistance on behalf of BioMarin Pharmaceutical Inc.

\section{Funding}

The writing of this manuscript was funded by BioMarin Pharmaceutical Inc.

\section{Availability of data and materials}

All data generated or analyzed during this study are included in this published article and its supplementary information files.

\section{Authors' contributions}

All authors contributed to design and helped to draft the manuscript. All authors read and approved the final manuscript.

\section{Competing interests}

Rudolf Schrover is owner of SYNEVi Pty Ltd and provided strategic advice to BioMarin on its application to the Pharmaceutical Benefits Advisory Committee (PBAC) seeking reimbursement for elosulfase alfa on the Life Saving Drug Programme (LSDP). Kathryn Evans is an employee of BioMarin Pharmaceutical Australia. Roberto Giugliani received travel grants to attend scientific meetings from Actelion, BioMarin, Genzyme, and Shire; speaker honoraria from Actelion, Amicus, BioMarin, Genzyme, PTC, and Shire; research and educational grants from Amicus, Actelion, Alexion, BioMarin, Genzyme, Shire, and Ultragenyx. Ian Noble is owner of Noble Pharma 
Consulting Pty LTD and provided strategic advice to BioMarin on its application to the PBAC seeking reimbursement for elosulfase alfa on the LSDP. Dr. Bhattacharya's department has received funding from BioMarin Pharmaceutical Inc. to participate in clinical trials. He has attended BioMarinsponsored meetings.

\section{Consent for publication}

Not applicable.

Ethics approval and consent to participate Not applicable.

\section{Publisher's Note}

Springer Nature remains neutral with regard to jurisdictional claims in published maps and institutional affiliations.

\begin{abstract}
Author details
'SYNEVi Pty Limited, Level 4, Suite 402, 15 Help Street, Chatswood NSW 2067, Australia. ${ }^{2}$ BioMarin Pharmaceutical Australia Pty Ltd, Sydney, NSW, Australia. ${ }^{3}$ Medical Genetics Service/HCPA, Department of Genetics/UFRGS and INAGEMP, Porto Alegre, Brazil. ${ }^{4}$ Noble Pharma Consulting, Sydney, NSW, Australia. ${ }^{5}$ Genetic Metabolic Disorders Service, Sydney Childrens' Hospital Network, Westmead, NSW, Australia. ${ }^{6}$ Discipline of Child and Adolescent Health, Sydney University, Sydney, NSW, Australia.
\end{abstract}

\section{Received: 10 January 2017 Accepted: 13 April 2017}

\section{Published online: 26 April 2017}

\section{References}

1. Hendriksz CJ, Lavery C, Coker M, Ucar SK, Jain M, Bell L, et al. Burden of disease in patients with Morquio A syndrome: results from an international patient-reported outcomes survey. Orphanet J Rare Dis. 2014;9:32.

2. Bank RA, Groener JE, van Gemund JJ, Maaswinkel PD, Hoeben KA, Schut HA, et al. Deficiency in $\mathrm{N}$-acetylgalactosamine-6-sulfate sulfatase results in collagen perturbations in cartilage of Morquio syndrome A patients. $\mathrm{Mo}$ Genet Metab. 2009;97:196-201.

3. Lavery C, Hendriksz C. Mortality in patients with Morquio Syndrome A. JIMD Rep. 2015;15:59-66.

4. Harmatz P, Mengel KE, Giugliani R, Valayannopoulos $V$, Lin SP, Parini R, et al. The Morquio A clinical assessment program: baseline results illustrating progressive, multisystemic clinical impairments in Morquio A subjects. Mol Genet Metab. 2013;109:54-61.

5. Montaño AM, Tomatsu S, Gottesman GS, Smith M, Orii T. International Morquio A registry: clinical manifestation and natural course of Morquio A disease. J Inherit Metab Dis. 2007;30:165-74.

6. Kakkis E, Neufeld E. The Mucopolysaccharidoses. 1996.

7. Hendriksz CJ, Harmatz P, Beck M, Jones S, Wood T, Lachman R, et al. Review of clinical presentation and diagnosis of mucopolysaccharidosis IVA. Mol Genet Metab. 2013;110:54-64

8. Hendriksz CJ, Berger Kl, Giugliani R, Harmatz P, Kampmann C, Mackenzie WG, et al. International guidelines for the management and treatment of Morquio A syndrome. Am J Med Genet A. 2015;167A:11-25.

9. John RM, Hunter D, Swanton RH. Echocardiographic abnormalities in type IV mucopolysaccharidosis. Arch Dis Child. 1990;65:746-9.

10. Hendriksz CJ, Burton B, Fleming TR, Harmatz P, Hughes D, Jones SA, et al. Efficacy and safety of enzyme replacement therapy with BMN 110 (elosulfase alfa) for Morquio A syndrome (mucopolysaccharidosis IVA): a phase 3 randomised placebo-controlled study. J Inherit Metab Dis. 2014;37: 979-90.

11. Society AT. ATS statement: guidelines for the six-minute walk test. Am J Respir Crit Care Med. 2002;166:111-7.

12. Li AM, Yin J, Au JT, So HK, Tsang T, Wong E, et al. Standard reference for the six-minute-walk test in healthy children aged 7 to 16 years. Am J Respir Crit Care Med. 2007:176:174-80.

13. Lammers AE, Hislop AA, Flynn Y, Haworth SG. The 6-minute walk test: normal values for children of 4-11 years of age. Arch Dis Child. 2008:93:464-8.

14. Ulrich S, Hildenbrand FF, Treder U, Fischler M, Keusch S, Speich R, et al. Reference values for the 6-minute walk test in healthy children and adolescents in Switzerland. BMC Pulm Med. 2013;13:49.
15. Harmatz PR, Mengel KE, Giugliani R, Valayannopoulos V, Lin SP, Parini R, et al. Longitudinal analysis of endurance and respiratory function from a natural history study of Morquio A syndrome. Mol Genet Metab. 2015;1 14:186-94.

16. Hendriksz CJ, Parini R, AlSayed MD, Raiman J, Giugliani R, Solano Villarreal $M L$, et al. Long-term endurance and safety of elosulfase alfa enzyme replacement therapy in patients with Morquio A syndrome. Mol Genet Metab. 2016;119:131-43.

17. Hendriksz CJ, Berger Kl, Parini R, AlSayed MD, Raiman J, Giugliani R, et al. Impact of long-term elosulfase alfa treatment on respiratory function in patients with Morquio A syndrome. J Inherit Metab Dis. 2016;39:839-47.

18. Hendriksz CJ, Parini R, AlSayed MD, Raiman J, Giugliani R, Mitchell JJ, Burton BK, Guelbert N, Stewart F, Hughes DA, Matousek R, Hawley SM, Decker C, Harmatz PR. Elosulfase alfa treatment and changes in physical functioning and disability in Morquio A. Presented at the World Symposium, 2017 (P140).

19. Lampe C, Jain M, Olaye A, Meesen B, Decker C,Mengel E. Relationship between patient-reported outcomes and clinical outcomes in patients with Morquio A syndrome. JIEMS. 2015;3:1-8.

20. Bittner V, Weiner DH, Yusuf S, Rogers WJ, McIntyre KM, Bangdiwala SI, et al. Prediction of mortality and morbidity with a 6-minute walk test in patients with left ventricular dysfunction. JAMA. 1993;270:1702-7.

21. Casanova C, Cote C, Marin JM, Pinto-Plata V, de Torres JP, Aguirre-Jaíme A, et al. Distance and oxygen desaturation during the 6-min walk test as predictors of long-term mortality in patients with COPD. Chest. 2008;134:746-52.

22. de Liefde II, van Domburg RT, Bax JJ, Klein J, Verhagen HJM, Poldermans D. A decline in walking distance predicts long-term outcome in patients with known or suspected peripheral artery disease. Eur J Cardiovasc Prev Rehabil. 2010;17:321-8.

23. Martin C, Chapron J, Hubert D, Kanaan R, Honoré I, Paillasseur JL, et al. Prognostic value of six minute walk test in cystic fibrosis adults. Respir Med. 2013;107:1881-7.

24. de Moraes KL, Ulisses Signori L, Antonini Ribeiro R, Vargas Silva AM, Moreira PR, Dipp $T$, et al. Prognostic value of the six-minute walk test in end-stage renal disease life expextancy: a prospective cohort study. Clinics. 2012;67:581-6.

25. Carey EJ, Steidley DE, Aqel BA, Byrne TJ, Mekeel KL, Rakela J, et al. Sixminute walk distance predicts mortality in liver transplant candidates. Liver Transpl. 2010:16:1373-8.

26. Golpe R, Castro-Añón O, Pérez-de-Llano LA, González-Juanatey C, MuñizFernandez C, Testa-Fernández A, et al. Prognostic significance of six-minute walk test in non-group 1 pulmonary hypertension. Heart Lung. 2014;43:72-6.

27. Jaeschke R, Singer J, Guyatt GH. Measurement of health status. Ascertaining the minimal clinically important difference. Control Clin Trials. 1989;10:407-15.

28. Henricson E, Abresch R, Han JJ, Nicorici A, Goude Keller E, de Bie E, et al. The 6-minute walk test and person-reported outcomes in boys with duchenne muscular dystrophy and typically developing controls: longitudinal comparisons and clinically-meaningful changes over one year. PLoS Curr. 2013:5.

29. du Bois RM, Albera C, Costabel U, Bradford WZ, Kartashov A, Noble PW et al. 6-minute walk test distance (6MWTD) is a reliable, valid, and responsive outcome measure that predicts mortality in patients with IPF. Am J Respir Crit Care Med. 2010;181:A1103.

30. du Bois RM, Weycker D, Albera C, Bradford WZ, Costabel U, Kartashov A, et al. Six-minute-walk test in idiopathic pulmonary fibrosis: test validation and minimal clinically important difference. Am J Respir Crit Care Med. 2011;183:1231-7.

31. Swigris JJ, Wamboldt FS, Behr J, du Bois RM, King Jr TE, Raghu G, et al. The six-minute walk in idiopathic pulmonary fibrosis: longitudinal changes and minimum important difference. Thorax. 2010;65:173-7.

32. Polkey MI, Spruit MA, Edwards LD, Watkins ML, Pinto-Plata V, Vestbo J, et al. Six-minute-walk test in chronic obstructive pulmonary disease: minimal clinically important difference for death or hospitalization. Am J Respir Crit Care Med. 2013;187:382-6.

33. Copay AG, Subach BR, Glassman SD, Polly Jr DW, Schuler TC. Understanding the minimum clinically important difference: a review of concepts and methods. Spine J. 2007;7:541-6.

34. McGlothlin AE, Lewis RJ. Minimal clinically important difference: defining what really matters to patients. JAMA. 2014;312:1342-3.

35. Henricson E, Abresch R, Han J, Nicorici A, Goude Keller E, de Bie E, et al. The 6-minute walk test and person-reported outcomes in boys with duchenne muscular dystrophy and typically developing controls: longitudinal comparisons and clinically-meaningful changes over one year. PLoS Curr. 2013;5. 
36. Lachmann R, Schoser B. The clinical relevance of outcomes used in lateonset Pompe disease: can we do better? Orphanet J Rare Dis. 2013;8:160.

37. Singh SJ, Puhan MA, Andrianopoulos V, Hernandes NA, Mitchell KE, Hill CJ, et al. An official systematic review of the European Respiratory Society/ American Thoracic Society: measurement properties of field walking tests in chronic respiratory disease. Eur Respir J. 2014;44:1447-78.

38. Wraith JE, Clarke LA, Beck M, Kolodny EH, Pastores GM, Muenzer J, et al. Enzyme replacement therapy for mucopolysaccharidosis I: a randomized, double-blinded, placebo-controlled, multinational study of recombinant human a-L-iduronidase (laronidase). J Pediatr. 2004;144:581-8.

39. Harmatz P, Giugliani R, Schwartz IVD, Guffon N, Teles EL, Miranda MCS, et al. Long-term follow-up of endurance and safety outcomes during enzyme replacement therapy for mucopolysaccharidosis VI: final results of three clinical studies of recombinant human $\mathrm{N}$-acetylgalactosamine 4-sulfatase. Mol Genet Metab. 2008:94:469-75.

40. Schlander M, Garattini S, Holm S, Kolominsky-Rabas P, Nord E, Persson U, et al. Incremental cost per quality-adjusted life year gained? The need for alternative methods to evaluate medical interventions for ultra-rare disorders. J Comp Eff Res. 2014;3:399-422.

41. Hendriksz CJ, Giugliani R, Harmatz P, Mengel E, Guffon N, Valayannopoulos $V$, et al. Multi-domain impact of elosufase alfa in Morquio $A$ syndrome in the pivotal phase III trial. Mol Genet Metab. 2015;114:178-85.

42. Holland AE, Hill CJ, Conron M, Munro P, McDonald CF. Small changes in sixminute walk distance are important in diffuse parenchymal lung disease. Respir Med. 2009;103:1430-5.

43. Holland AE, Hill CJ, Rasekaba T, Lee A, Naughton MT, McDonald CF. Updating the minimal important difference for six-minute walk distance in patients with chronic obstructive pulmonary disease. Arch Phys Med Rehabil. 2010;91:221-5.

44. Puhan MA, Chandra D, Mosenifar Z, Ries A, Make B, Hansel NN, et al. The minimal important difference of exercise tests in severe COPD. Eur Respir J. 2011;37:784-90.

45. Redelmeier DA, Bayoumi AM, Goldstein RS, Guyatt GH. Interpreting small differences in functional status: the six minute walk test in chronic lung disease patients. Am J Respir Crit Care Med. 1997:155:1278-82.

46. Lee AL, Hill CJ, Cecins N, Jenkins S, McDonald CF, Burge AT, et al. Minimal important difference in field walking tests in non-cystic fibrosis bronchiectasis following exercise training. Respir Med. 2014;108:1303-9.

47. Gremeaux V, Troisgros O, Benaïm S, Hannequin A, Laurent Y, Casillas J-M, Benaïm C. Using field walk tests to individualize exercise training in coronary artery disease patients. Arch Phys Med Rehabil. 2011;92:1731. Abs. 137.

48. Gremeaux V, Troisgros O, Benaïm S, Hannequin A, Laurent Y, Casillas JM, et al. Determining the minimal clinically important difference for the sixminute walk test and the 200-meter fast-walk test during cardiac rehabilitation program in coronary artery disease patients after acute coronary syndrome. Arch Phys Med Rehabil. 2011;92:611-9.

49. Mathai SC, Puhan MA, Lam D, Wise RA. The minimal important difference in the 6-minute walk test for patients with pulmonary arterial hypertension. Am J Respir Crit Care Med. 2012;186:428-33.

50. Kwok BC, Pua YH, Mamun K, Wong WP. The minimal clinically important difference of six-minute walk in Asian older adults. BMC Geriatr. 2013;13:23.

51. Puhan MA, Mador MJ, Held U, Goldstein R, Guyatt GH, Schünemann HJ. Interpretation of treatment changes in 6-minute walk distance in patients with COPD. Eur Respir J. 2008;32:637-43.

52. Wise RA, Brown CD. Minimal clinically important differences in the sixminute walk test and the incremental shuttle walking test. COPD. 2005;2: $125-9$.

53. Gilbert C, Brown MC, Cappelleri JC, Carlsson M, McKenna SP. Estimating a minimally important difference in pulmonary arterial hypertension following treatment with sildenafil. Chest. 2009;135:137-42.

54. McDonald CM, Henricson EK, Abresch RT, Florence J, Eagle M, Gappmaier E, et al. The 6-minute walk test and other clinical endpoints in duchenne muscular dystrophy: reliability, concurrent validity, and minimal clinically important differences from a multicenter study. Muscle Nerve. 2013;48:357-68.

55. Taeger T, Wians FH, Cebola R, Froehlich H, Katus HA, Frankenstein L, et al. Biovaribility, minimal important difference and change of the 6 -minute walk test in chronic heart failure. Eur J Heart Failure Suppl. 2013;S94:P820.

\section{Submit your next manuscript to BioMed Central and we will help you at every step:}

- We accept pre-submission inquiries

- Our selector tool helps you to find the most relevant journal

- We provide round the clock customer support

- Convenient online submission

- Thorough peer review

- Inclusion in PubMed and all major indexing services

- Maximum visibility for your research

Submit your manuscript at www.biomedcentral.com/submit
Biomed Central 